\title{
Practice Research on the Subject Knowledge Platform Based on the Knowledge Architecture
}

\author{
Rui Shao ${ }^{1, a^{*},}$ Yu-qiang Guo, b, Xian-hua Fu ${ }^{1, c}$ and Shi-ming Zhong ${ }^{2, d}$ \\ ${ }^{1}$ Library, Wuhan University of Technology, Wuhan, P.R.China \\ ${ }^{2}$ Institute of Geodesy and Geophysics, Chinese Academy of Sciences, Wuhan, P.R.China \\ asorrny@whut.edu.cn, b464139572@qq.com, cxhfu@whut.edu.cn, d287040415@qq.com
}

Keywords: Subject knowledge, Knowledge architecture, Practice platform, Sharing platform

\begin{abstract}
On the basis of knowledge architecture theories, the author put forward to elaborate the construction of the subject knowledge platform from perspectives of resource construction, systematic framework and service function. By strengthening construction of internet academic resources, expert academic resources and expanded academic resources, an academic sharing space is built where subject librarians and scientific research users can commonly participate in, mutually interact and share knowledge with each other. A platform is established for information exchange and sharing, and provide personalized customization of subject resources and RSS push services so as to realize the acquisition, organization, mining, development and innovation of subject knowledge. A service platform is provided for scientific research users with multiple-service customization and multiple-resource integration.
\end{abstract}

\section{Introduction}

Under the digital research environment, knowledge spreads through the network mainly in the digital form and structures of knowledge are more and more complicated. With more and more digital library resources provided by social information institutions, service concepts of libraries change fundamentally due to the development of academic search engine technologies represented by Baidu Academic Search [1], and traditional library lending service and information reference and consultation service is greatly reduced compared with previous days. To satisfy changed demands of teaching and scientific research users for information exchanges and knowledge sharing, subject service of research libraries has gradually developed into integration, profession, service subjectification and service socialization. Therefore, university libraries must strengthen the construction of subject knowledge platforms [2].

The knowledge architecture is a higher-level form of resource construction based on information construction [3], which realizes a deeper level knowledge service by combining the framework of information construction system and knowledge element indexing and linking technologies to provide users with services of knowledge navigation, retrieval, recombination and integration [4]. The knowledge architecture can effectively guarantee the knowledge discovery and acquisition capability, knowledge organization and integration capability and knowledge development and utilization capability of digital libraries so as to effectively realize their knowledge service capability. With the rapid development of advanced network technologies, various wireless communication technologies and knowledge architecture theories which provide technological and theoretical supports for constructing centralized information systems, this paper puts forward to conduct researches on practical work of new subject knowledge platforms based on the knowledge architecture under this situation, hoping to improve discipline services of university libraries.

\section{Optimizing Functions of the Knowledge Architecture on Subject Knowledge Services of University Libraries}

The knowledge architecture can focus on users' personalized information, make knowledge products well integrated into users' work environment and promote users' dynamic exchanges and 
optimize subject services. From the perspective of content construction, the knowledge architecture emphasizes the conversion from information to knowledge, the extraction and indexing of knowledge elements, the forming of knowledge networks based on knowledge element links, the application of semantic networks, classifications and integrations of subject knowledge structures and the construction of subject knowledge warehouse to create a complete subject knowledge environment. From the perspective of the subject resource organization, the knowledge architecture, taking "scientific research users" as the center, uniformly manages and integrates subject resources, effectively meeting diversified service demands of different users and showing the diversification, humanization and intellectualization of discipline services. From the perspective of the service form, the knowledge architecture focuses on providing personalized discipline services for scientific research users and making knowledge products better integrated into users' research environment, which is good for promoting exchanges and dynamic interactions among subject librarians and scientific research users and satisfies scientific research users' demands for information and sharing and co-building of subject information resources. From the perspective of the service content, the knowledge architecture focuses on converting subject information to knowledge, reusing knowledge and guiding users to master subject knowledge systems so as to acquire subject knowledge they need, gradually convert implicit knowledge to explicit knowledge, promote knowledge sharing and realize knowledge innovation. To sum up, the construction of subject knowledge platform based on the knowledge architecture can effectively deepen the development of subject knowledge services in service practices.

\section{Strategies on Resource Construction of the Subject Knowledge Platform}

For scientific research users, the subject knowledge platform can save their time to select resources to the maximum, so they can efficiently acquire professional academic resources relevant to their research fields to meet their demands for information resources. The subject knowledge platform can effectively solve the contradiction that academic users hope to rapidly acquire resources and resources grow continuously in a disorderly manner [5]. Therefore, the construction of information resources is very important for the construction of the subject knowledge platform. The platform must have a large variety of well-organized, highly professional and high-quality information resources. Considering the construction requirements of key disciplines of schools and development goals of the library, in 2014, the library where the author works started to reconstruct subject knowledge services, integrate thematic database resources of four subjects including "Information and Communication Engineering, Naval Architecture and Ocean Engineering, Control Science and Engineering and Mechanical Engineering", and allocate subject resources according to internet academic resources, expert academic resources and expanded academic resources on the basis of demands of research service objects, so as to realize the integration of subject resources.

3.1. Strategies on construction of internet academic resources. Scientifically selecting mass internet resources is the key to resource construction of subject platforms. In July 2006, Harvard University Library [6] launched the "WAX Project", which was a digital resource system specially developed based on internet and was officially put into use in February 2009. Librarians of Harvard University Library provide applicants through the system with inquiry and storage services of internet resources of academic values. Referring to "WAX Project", the key to select mass internet information resources is that libraries should gather academic networks such as authoritative academic organizations, associations, subject expert networks, domestic and foreign subject institution network and academic blogs as sources of academic dynamic resources so as to guarantee literature resources to be high-quality, strongly authoritative and professional.

The construction of internet resources should focus on domestic and foreign authoritative experts and institutions in relevant subject research fields so as to acquire high-quality literature materials about subject development such as literature reviews, hotspot technological materials, results of subject fields and advanced technological information. Internet resources collected through web crawler technology [7] based on topic searches should be selected carefully by subject librarians who 
should seek for professional and refined academic resources, have specific objectives, and guarantee values of long-term collections. Construction procedures of internet academic resources are as shown in Figures 1:

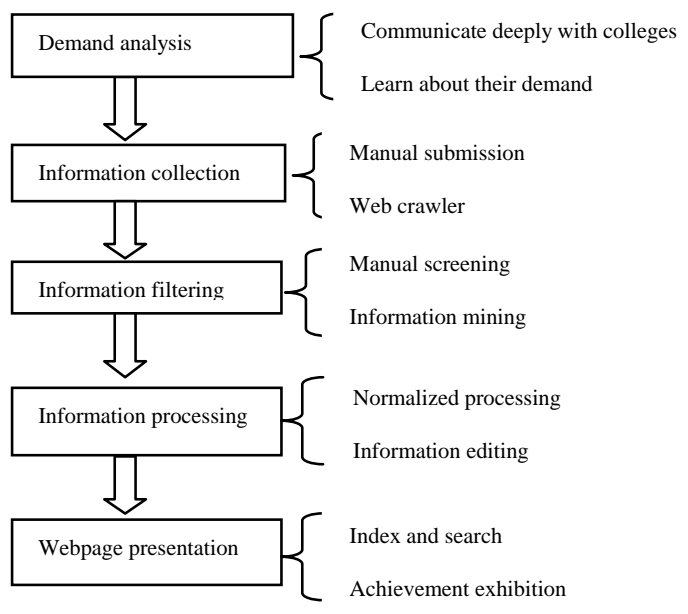

Fig. 1 Construction Procedures of Internet Academic Resources

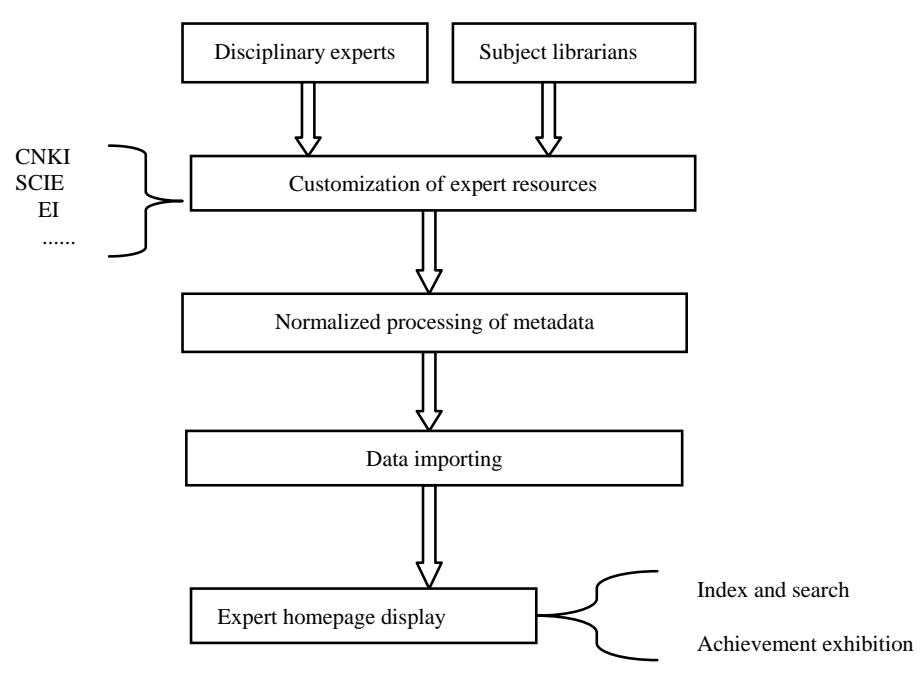

Fig. 2 Construction Procedures of Expert Academic Resources

3.2. Strategies on construction of expert academic resources. Because implicit knowledge is hard to be captured and acquired, the externalization of implicit knowledge is a behavior of knowledge management and certain tools must be used to achieve the sharing of implicit knowledge [8]. Experts and scholars have certain implicit knowledge. The academic sharing space built in the subject knowledge platform makes it possible for experts and scholars to exchange their implicit knowledge. Through the expert customization function in the academic sharing space, subject librarians can guide experts to sort out their research literatures, strengthen the management of research results and research information resources, which can guarantee the accuracy of renewed academic resource data. Experts can upload materials, information and achievements of research topics to the academic sharing space all by themselves or asking helps from subject librarians so as to improve their personal interests in displaying their academic achievements. By integrating experts' subject knowledge information, academic achievements in the academic sharing space will be finally displayed on Author Profile Pages (APP for short).

APP [9] is a navigation platform of information resources taking subject experts as the center, which finds out and organizes subject expert knowledge taking expert subject knowledge chain as the mainline. The platform introduces in details research content, academic achievements and ongoing projects in relevant research fields of all experts, and uploads lists of published literatures, literature information and information about cooperative experts in chronological order. Literature information resources of experts provided by the platform have not only high values but also close relations with subject research directions, which can help teaching and scientific research users master forefront information in relevant research fields. To guarantee the novelty of literature information resources, subject librarians must update expert information at regular intervals. Construction procedures of expert academic resources are as shown in Figures 2.

3.3. Strategies on construction of expanded academic resources. The library where the author works has formed a subject service team at the beginning of constructing the subject knowledge platform. By using various evaluation tools including ESI and Thomson Data Analyzer and taking their advantages in subject analyses and predictions, the subject knowledge group of the team statistically analyzes and sorts subject research institutions, comprehensively analyzes the history and trends of the subject development, and provides detailed subject evaluations through the subject knowledge platform. Their efforts are mainly demonstrated in the subject research and teaching achievement measurements by carefully and thoroughly analyzing and comparing data, the document analyses and citation analyses on the information subject; the analysis of subject cited journals. To make results of data analyses accurate and reliable and subject evaluations valuable references, the 
subject knowledge platform provides subject evaluations with the following functions: (1) Conduct subject contrastive analyses on professional evaluation-type databases purchased by libraries to avoid incomplete and non-authoritative analysis results caused by a single database; (2) Make targeted and personalized analyses according to demands of users to guarantee the practicability of analysis results of subject evaluations; (3) Take disciplines as research objects of subject services and evaluate research achievements of the disciplines so as to find out an evaluation mode applicable to all subject research results of this school.

\section{Construction of Subject Knowledge Platform}

Under the ubiquitous knowledge environment, subject knowledge services take scientific research users as the center and subjects as the unit and fully integrate into users' virtual spaces, realize the individuation, professionalization and dynamism of subject services, conduct the collection and sort-out of information and the acquisition and innovation of knowledge according to demands of scientific research users, and integrate subject knowledge services into the process of knowledge acquisition, innovation and sharing, through mutual interactions of subject service teams and scientific research users who jointly participate in resource construction of the subject knowledge platform.

Based on the needs of scientific research users for knowledge services, the author puts forward to focus on the direction of scientific researches, mine subject knowledge contained in internet information resources, technological literature and meeting information, construct an academic sharing space where subject librarians jointly participate in, mutually interact and share knowledge with each other. A platform is built for information sharing and exchange, provides personalized customization of subject resources and RSS push services so as to realize the acquisition, organization, mining, development and innovation of subject knowledge, provide scientific research users with subject knowledge services with multiple-service customization and multiple-resource integration.

4.1. System architecture of the subject knowledge platform. The system mainly includes several core elements such as subject service team, scientific research users, academic sharing spaces, expert databases and academic resource navigation. Subject service team is responsible for collecting, sorting out and organizing internet academic resources. Subject service team and scientific research users realize real-time online communication through instant messaging tools and realize synchronous communication and jointly construct expert knowledge databases through academic sharing spaces. Subject service team is responsible for the construction of expanded academic resources. Through academic sharing spaces, scientific research users make feedbacks and suggestions, interact and communicate with subject librarians. They can upload report materials, information and achievements of their research topics to expert knowledge database for management. All above-mentioned subject information and resources are organized orderly through the subject knowledge platform which is beneficial to utilizing and sharing knowledge. Subject service teams and scientific research users can not only interact and cooperate in the internet virtual environments, but also cooperate and communicate face to face in discussion rooms of libraries. Research sharing spaces are composed of virtual academic sharing spaces and spaces in real world.

4.2. Implementation methods of the subject knowledge platform. The library of The University of North Carolina at Greensboro (NUCG for short) pointed out in its research report that libraries must meet space demands of experts and scholars by providing them with virtual space services [10] and constructing academic sharing spaces so as to better serve scientific researches. The subject knowledge platform builds subsystems such as academic sharing spaces, expert database, instance messaging systems and internet academic database and then it effectively integrates all subsystems by using Ajax Technology to realize one-stop navigation with regard to subject resources of libraries. The subject knowledge platform perfectly integrates special services of all systems and continuously expands functions of the platform. Through the platform, scientific research users can acquire 
complete subject resources, share, mine knowledge, communicate and cooperate with each other, and dynamically interact with subject librarians.

Academic sharing spaces: Academic sharing spaces are realized by adopting Ajax Technology. Feedbacks and suggestions are collected by using demand forms of information and services. Build platforms for information sharing and exchanges to make it possible for experts and subject librarians to interact and communicate, learn about real-time experts' research topics, information demands and utilizations of information resources of the platform, and thoroughly find out explicit and implicit demands of subject information services [11]. Through good information feedback systems, we can learn about people and information resources which experts pay attention to in scientific research fields of certain disciplines so as to indirectly guide subject librarians to construct the subject knowledge platform, guaranteeing the quality of information resource construction.

Expert database: Expert database is realized based on APP. The data sources of APP are based on resources of library database of this library. And APP adopts DOI technology to guarantee the long-term effectiveness and novelty of links to original literatures. Through link addresses of literatures provided by authoritative experts, it is convenient to conduct research on relevant disciplines. Meanwhile, by clicking the item of academic achievements and ongoing research projects on the page, people can get information on all academic achievements in the current year or lists of projects so as to achieve the organization of subject knowledge. The information list of cooperative experts can show social relations among experts and therefore scientific research scholars can acquire more relevant academic information resources.

Instant messaging systems: Instant messaging systems are realized based on the software called QQ. The software has good universality, good performance and concise interface, and offers functions including video conference, voice conversation and text chatting. The software has a vast number of users, so it is applicable to instant communication between scientific research users and subject librarians and makes real-time interactions convenient.

Information fusion: Information fusion is realized based on IIS+ASP. Support fusion standards of RSS. Provide services of automatic fusions, update, push and post of information. Provide user-driven updating mechanism which automatically updates in the background. Provide functions such as multi-level catalog management, combinatorial search and library database management. Ajax Technology is adopted to show resource lists and recognize aggregation sources. Subject librarians extensively collect online information sources and gather them together according to disciplines and allow scientific research users to add addresses of aggregation sources anytime which will be included into the aggression system after examination, verification and classification to fully play roles of the co-construction mechanism and provide value-added services for scientific research uses.

Subject resource navigation homepage: The subject resource navigation homepage is realized based on Ajax Technology. Combine Ajax Technology with programming techniques such as Java, XML and JavaScript to integrate above-mentioned several subsystems into the he subject resource navigation homepage, all columns of which can be unfolded, expanded, dragged and rearranged and can provide personalized academic resource customization according to demands of scientific research users. Academic resource customization means users can make personalized resource subscriptions according to their research demand and knowledge structure. All thematic academic resources of the platform are provided to scientific research users to conduct resource customization by checking web forms. Scientific research users can check resource columns which they are interested in. Thematic resources stored will be updated automatically with the update of platform resources.

\section{Summary}

The subject knowledge platform achieves the integration of subject resources. It is necessary for scientific research users to know about values of the subject knowledge platform and effectively use its academic information resources. Expanded and improved subject knowledge platform not only 
displays a large variety of orderly organized, highly professional and high-quality information resources, but also has expanded content, including academic resources such as scientific research trends, academic achievements and grey literatures related to experts and scholars, providing conveniences for teaching and scientific research personnel, school managers and other school departments to acquire academic resources. The construction of academic sharing spaces develops the subject knowledge platform into an "exchange platform" instead of an "academic platform", which promotes activities of knowledge exchanges of this school. Functions of the subject knowledge platform are continuously expanded and developed. The next step is to provide more value-added services applied in scientific research management by developing and updating systems and gradually develop the subject knowledge platform into a school-wide academic exchange center.

\section{Acknowledgment}

This work is supported by Library and Information Committee for Academic Libraries of Hubei Province: Research on Service Exploration of Subject Service Platform. All support is gratefully acknowledged.

\section{References}

[1] Information on http://xueshu.baidu.com.

[2] Long Yanjun, Zhou Fangdu, A Study on the Subject Service Pattern and Service Platform of Library in China, Library. 5 (2013) 126-128.

[3] Liu Wenke, Research on the Website Construction of University Library Based on the Idea of Knowledge Architecture, The Journal of the Library Science in Jiangxi. 2 (2008) 11-17.

[4] Si Li, Realization of knowledge service based on knowledge architecture, Library Tribune. 6 (2009) 216-219.

[5] Liu Xiuhua, Wen Xiaoming, Xiao Jianping, The Investigation and Analysis of the Current Development Situation and Transformation of "985" Universities' Discipline Navigation, Library and Information Service. 20 (2014) 55-60.

[6] Information on http:// wax.lib.harvard.edu/collections/about.do?kind=about\&lang=eng/.

[7] Yu Juan, Liu Qiang, Survey on Topic-focused Crawlers, Computer Engineering \& Science. 2 (2015) 231-237.

[8] Hu Renfeng, Liu Guoliang, An Empirical Research of Industry-university Collaborative Innovation Tacit Knowledge Sharing Factors in Mobile Internet Environment, Library and Information Service. 7 (2015) 48-54.

[9] Information on http://www.acm.org/publications/acm-author-profile-page/.

[10]Qiang Jing, Huang Fuming, Study on the Space Evaluation of the University Library in America and Its Enlightenments, Library Development. 2 (2015) 92-95.

[11]Wang Xue, Research on the Practice of the Subject Information Service Embedded in the Scientific Research Process, Library Development. 4 (2015) 83-86. 